\title{
Epistemologia sobre amputações e desbridamentos de membros inferiores realizados no Hospital Universitário de Maringá
}

\author{
Epistemology of lower limb amputations and debridements at \\ Hospital Universitário de Maringá
}

\author{
Amélia Cristina Seidel ${ }^{1}$, Andréia K. Nagata ${ }^{2}$, Hemerli C. de Almeida ${ }^{3}$, Márcia Bonomo ${ }^{3}$
}

\section{Resumo}

Contexto: Não há estatísticas precisas sobre o número de amputações realizadas anualmente, mas é conhecido o comprometimento da qualidade de vida desses indivíduos e a necessidade de uma equipe multiprofissional para sua reabilitação.

Objetivo: Analisar todas as amputações de membros inferiores realizadas pelo Serviço de Angiologia e Cirurgia Vascular do Hospital Universitário Regional de Maringá entre 2000 e 2006.

Métodos: Estudo descritivo e retrospectivo a partir dos prontuários de pacientes submetidos a amputação ou desbridamento cirúrgico de membros inferiores por trauma ou doença vascular.

Resultados: Ocorreram 116 procedimentos, sendo $84,5 \%$ amputações e $15 \%$ desbridamentos cirúrgicos, em 111 pacientes, sendo 78 homens e 33 mulheres, cuja média das idades foi de 63,4 anos ( 4 a 100 anos), sendo os principais fatores etiológicos: doenças vasculares $(92,8 \%)$ e trauma $(7,2 \%)$. As comorbidades e fatores de risco mais prevalentes foram: hipertensão arterial sistêmica $(66 \%)$, diabetes melito $(60 \%)$, tabaco $(28 \%)$ e arritmia cardíaca $(0,9 \%)$. Quanto à escolaridade, se observou que $52,2 \%$ dos pacientes cursaram apenas o ensino fundamental, $6,5 \%$ fizeram o ensino médio, completo ou incompleto, e $41,3 \%$ eram analfabetos. A percentagem de pacientes submetidos à amputação primária foi de $94,9 \%$, e secundárias, $5,1 \%$. No entanto, houve diminuição progressiva do coeficiente desses procedimentos a cada ano.

Conclusões: Este trabalho permite inferir que a incidência de amputação de membros inferiores foi maior no sexo masculino e teve como principal causa a doença aterosclerótica, mas apresentou uma redução progressiva.

Palavras-chave: Amputação, membros inferiores, doenças vasculares, doenças vasculares diabéticas, traumatismos.

\begin{abstract}
Background: There are no precise data on the number of amputations performed every year. However, the decrease in quality of life of patients submitted to amputation is well-known, as is the need to involve a multidisciplinary team in the rehabilitation of these individuals.
\end{abstract}

Objective: To analyze all lower limb amputations performed by the Angiology and Vascular Surgery at Hospital Universitário Regional de Maringá, from 2000 to 2006.

Methods: A descriptive and retrospective study was performed to review medical records of patients submitted to amputation or surgical debridement of lower limbs to treat vascular diseases or trauma.

Results: A total of 116 procedures were carried out $(84.5 \%$ amputations and $15 \%$ debridements) in 111 patients; 78 were males and 33 were females, with a mean age of 63.4 years (4-100 years). The main etiological factors were vascular diseases $(92.8 \%)$ and trauma $(7.2 \%)$. The most prevalent comorbidities and risk factors were hypertension $(66 \%)$, diabetes mellitus $(60 \%)$, tobacco $(28 \%)$ and cardiac arrhythmia $(0.9 \%)$. As to schooling, $52.2 \%$ of the patients had complete elementary school, $6.5 \%$ had complete or incomplete high school, and $41.3 \%$ were illiterate. Primary amputation was performed in $94.9 \%$ of patients, and secondary amputation in $5.1 \%$. However, there was a progressive reduction in amputation rate at each year.

Conclusions: Incidence of lower limb amputation was greater in males, had atherosclerotic disease as its main cause, but had progressive reduction.

Keywords: Amputation, lower limbs, vascular disease, diabetic vascular disease, trauma.

1. Doutora, Universidade Federal de São Paulo - Escola Paulista de Medicina (UNIFESP-EPM), São Paulo, SP. Professora adjunta, Angiologia e Cirurgia Vascular, Universidade Estadual de Maringá (UEM), Maringá, PR. Ultra-sonografista vascular e especialista em Cirurgia Cardiovascular, SBACV e Colégio Brasileiro de Radiologia (CBR).

2. Médica, UEM, Maringá, PR.

3. Acadêmico(a) de Medicina (6ํano), UEM, Maringá, PR.

Trabalho realizado na disciplina de Angiologia e Cirurgia Vascular, Curso de Medicina, Universidade Estadual de Maringá (UEM), Maringá, PR.

Apresentado como pôster no II Congresso Internacional de Saúde e no VI Seminário Científico do Centro de Ciência da Saúde da UEM, realizado em Maringá, em outubro de 2007.

Não foram declarados conflitos de interesse associados à publicação deste artigo.

Artigo submetido em 07.10.07, aceito em 28.08.08.

J Vasc Bras. 2008;7(4):308-315.

Copyright@ 2008 by Sociedade Brasileira de Angiologia e de Cirurgia Vascular 


\section{Introdução}

Atribuem-se, atualmente, à doença aterosclerótica obliterante periférica (DAOP) e ao diabetes melito (DM) os principais fatores relacionados às amputações de membros inferiores (MMII) ou membros superiores ${ }^{1-6}$. No entanto, o trauma já é uma expressiva causa de amputações periféricas, principalmente em pacientes jovens $^{7-14}$.

O exame atento da extremidade e a investigação do grau de comprometimento arterial ditarão o nível adequado para realização da amputação, que deve preservar o maior segmento possível do membro, visando a uma melhor recuperação do paciente ${ }^{15}$. A infecção comumente associada à gangrena é comum em diabéticos e merece tratamento adequado, evitando-se uma má evolução ${ }^{1,16}$.

Notório se faz, ainda, o tratamento adequado das lesões tróficas infectadas, que devem ser abordadas de forma multidisciplinar e freqüentemente associadas a diversas modalidades terapêuticas, possibilitando, assim, reduzir a proliferação de microorganismos de caráter patogênico, tendo papel coadjuvante as revascularizações, desbridamentos, uso de antibióticos e a oxigenoterapia hiperbárica ${ }^{17-19}$, que devem, no entanto, ser instituídos o mais precoce possível e nunca isoladamente ${ }^{8}$.

De maneira semelhante, o diagnóstico precoce nos casos de traumatismos vasculares se revela de extrema importância, particularmente na vigência de lesões graves e complexas. No entanto, a amputação muitas vezes se faz necessária e se mostra como procedimento decisivo para salvar a vida do doente e melhorar sua qualidade de vida ${ }^{1,13,17}$.

Diante do exposto, realizou-se este estudo, cujo objetivo foi analisar todas as amputações de MMII realizadas exclusivamente pelo Serviço de Angiologia e Cirurgia Vascular do Hospital Universitário Regional de Maringá (HUM) entre os anos de 2000 e 2006.

\section{Método}

Trata-se de um estudo retrospectivo de coorte, descritivo e analítico, que foi realizado após aprovação pelo Comitê de Ética da Universidade Estadual de Maringá a partir dos prontuários de pacientes submetidos a amputação e/ou desbridamento de MMII, entre janeiro de 2000 e janeiro de 2006, levando-se em consideração: idade, sexo, escolaridade, comorbidades e fatores de risco, além dos métodos propedêuticos realizados. Todos os pacientes foram incluídos.

A análise descritiva levou em consideração todas as variáveis supracitadas. Já nas análises quantitativas foram utilizados os testes qui-quadrado ou exato de Fisher no caso da avaliação de homogeneidade dos dados ou ainda pela observação dos valores mínimos e máximos e do cálculo de médias e desvio padrão. O nível de significância previamente definido para se rejeitar a hipótese de nulidade foi de $5 \%(\mathrm{p}<0,05)$ quando aplicável.

\section{Resultados}

Durante o período, foram analisados 116 procedimentos, provenientes de 111 pacientes, cuja média de idade foi de 63,4 anos (4 a 100 anos) e mediana de 65 anos. A grande maioria (78 pacientes) era do sexo masculino, e 33, do sexo feminino (relação 2,4:1). No que diz respeito às amputações, estas foram subdivididas da seguinte maneira: 98 (84,5\%) amputações $(37,7 \%$ transfemorais; $23,5 \%$ transtibiais; e $38,8 \%$ nos pés) e 18 $(15,5 \%)$ casos de desbridamentos cirúrgicos.

A estratificação dos pacientes por idade revelou que 17,1\% tinham menos de 50 anos; $46,8 \%$ tinham idade entre 51 anos e 70 anos; e 36\% tinham mais de 70 anos.

Quanto à escolaridade, 52,2\% tinham apenas cursado até o ensino fundamental, 6,5\% tinham ensino médio completo ou incompleto, e 41,3\% eram analfabetos (Tabela 1).

Os principais fatores etiológicos foram: trauma, oito casos $(7,2 \%)$; e doenças vasculares, 103 casos $(92,8 \%)$, sendo $99 \%$ em conseqüência da DAOP e $1 \%$ por doença tromboembólica. As comorbidades mais prevalentes nos casos de amputações devido às doenças vasculares foram: a hipertensão arterial sistêmica (HAS) (66\%), o DM $(60 \%)$, o tabagismo (59\%), a insuficiência renal $(11 \%)$ e a arritmia cardíaca $(0,9 \%)$, sendo esta responsável pela amputação transfemoral em um homem de 92 anos de idade como conseqüência da obstrução embólica da artéria ilíaca externa (Tabelas 2, 3 e 4). 
Tabela 1 - Distribuição de freqüência dos pacientes segundo sexo e escolaridade

\begin{tabular}{|c|c|c|c|c|c|c|}
\hline \multirow[b]{3}{*}{ Escolaridade } & \multicolumn{4}{|c|}{ Sexo } & \multicolumn{2}{|c|}{ Total } \\
\hline & \multicolumn{2}{|c|}{ Masculino } & \multicolumn{2}{|c|}{ Feminino } & \multirow[b]{2}{*}{$\mathbf{n}$} & \multirow[b]{2}{*}{$\%$} \\
\hline & $\mathbf{n}$ & $\%$ & $\mathbf{n}$ & $\%$ & & \\
\hline $\begin{array}{l}\text { Ensino } \\
\text { fundamental }\end{array}$ & 19 & 57,6 & 5 & 38,5 & 24 & 52,2 \\
\hline Ensino médio & 3 & 9,0 & 0 & 0,0 & 3 & 6,5 \\
\hline Analfabetos & 11 & 33,3 & 8 & 61,5 & 19 & 41,3 \\
\hline Total & 33 & 100 & 13 & 100 & 46 & 100 \\
\hline
\end{tabular}

Valores não observados $=65$.

Teste exato de Fisher $(\mathrm{p}=0,177)$.

Tabela 2 - Distribuição de freqüência dos pacientes segundo procedimento e diabetes melito

\begin{tabular}{|c|c|c|c|c|c|c|}
\hline \multirow[b]{3}{*}{ DM } & \multicolumn{4}{|c|}{ Procedimento } & \multicolumn{2}{|c|}{ Total } \\
\hline & \multicolumn{2}{|c|}{ Desbridamento } & \multicolumn{2}{|c|}{ Amputação } & \multirow[b]{2}{*}{$\mathbf{n}$} & \multirow[b]{2}{*}{$\%$} \\
\hline & $\mathbf{n}$ & $\%$ & $\mathbf{n}$ & $\%$ & & \\
\hline Não & 6 & 13 & 40 & 87 & 46 & 39,65 \\
\hline Sim & 12 & 17,1 & 58 & 82,9 & 70 & 60,35 \\
\hline Total & 18 & 15,5 & 98 & 84,5 & 116 & 100 \\
\hline
\end{tabular}

$\mathrm{DM}=$ diabetes melito.

Teste qui-quadrado $(\mathrm{p}=0,551)$.

A percentagem de pacientes submetidos à amputação primária foi de $94,9 \%$, e as amputações secundárias ocorreram em 5,1\% dos casos, devido principalmente a trombose e infecção.

Por outro lado, observou-se diminuição progressiva anual do coeficiente das amputações devido a DAOP, já que $38,8 \%$ dos casos ocorreram entre 2000 e $2003 ; 35,7 \%$, entre 2003 e 2004; $22,4 \%$, entre 2004 e 2005; e apenas $3,1 \%$ ocorreram entre 2005 e 2006.

Os casos de amputações devido a trauma de MMII foram realizados por danos irreversíveis na extremidade sem condições de revascularização como medida de prevenção da amputação. Predominaram nos homens
$(87,5 \%)$ jovens (média das idades de 30,7 anos - 4 a 60 anos). Foram realizadas três amputações transfemorais $(37,5 \%)$, uma transtibial $(12,5 \%)$ e quatro no pé $(50 \%)$. Somente em dois casos de amputação transfemoral foi pedido creatinina (3,3 e $0,68 \mathrm{mg} / \mathrm{dL}$, respectivamente); no restante dos casos desse grupo, não havia outro exame complementar solicitado.

Em relação à propedêutica pré-operatória dos pacientes com doença vascular não-traumática, 14 $(13,6 \%)$ foram submetidos a angiografia, e em apenas três $(2,9 \%)$ casos empregou-se o ultra-som vascular com Doppler.

Neste grupo, foram realizados $18(16,6 \%)$ desbridamentos e $90(83,3 \%)$ amputações. De acordo com o local 
Tabela 3 - Distribuição de freqüência dos pacientes segundo procedimento e hipertensão arterial sistêmica

\begin{tabular}{|c|c|c|c|c|c|c|}
\hline \multirow[b]{3}{*}{ HAS } & \multicolumn{4}{|c|}{ Procedimento } & \multicolumn{2}{|c|}{ Total } \\
\hline & \multicolumn{2}{|c|}{ Desbridamento } & \multicolumn{2}{|c|}{ Amputação } & \multirow[b]{2}{*}{$\mathbf{n}$} & \multirow[b]{2}{*}{$\%$} \\
\hline & $\mathbf{n}$ & $\%$ & $\mathbf{n}$ & $\%$ & & \\
\hline Não & 8 & 19,5 & 33 & 80,5 & 41 & 35,4 \\
\hline Sim & 10 & 13,3 & 65 & 86,7 & 75 & 64,6 \\
\hline Total & 18 & 15,5 & 98 & 84,5 & 116 & 100 \\
\hline
\end{tabular}

HAS = hipertensão arterial sistêmica.

Teste qui-quadrado $(\mathrm{p}=0,380)$.

Tabela 4 - Distribuição de freqüência dos pacientes segundo procedimento e tabagismo

\begin{tabular}{|c|c|c|c|c|c|c|}
\hline \multirow[b]{3}{*}{ Tabagismo } & \multicolumn{4}{|c|}{ Procedimento } & \multicolumn{2}{|c|}{ Total } \\
\hline & \multicolumn{2}{|c|}{ Desbridamento } & \multicolumn{2}{|c|}{ Amputação } & \multirow[b]{2}{*}{$\mathbf{n}$} & \multirow[b]{2}{*}{$\%$} \\
\hline & $\mathbf{n}$ & $\%$ & $\mathbf{n}$ & $\%$ & & \\
\hline Não & 5 & 15 & 29 & 85 & 34 & 39 \\
\hline Sim & 4 & 12,2 & 29 & 87,8 & 33 & 38 \\
\hline Ex-fumante & 3 & 15 & 17 & 85 & 20 & 23 \\
\hline Total & 12 & 13,8 & 75 & 86,2 & 87 & 100 \\
\hline
\end{tabular}

Valores não observados $=26$.

Teste exato de Fisher $(\mathrm{p}=1,000)$.

dos procedimentos, houve $34(37,8 \%)$ transfemorais, 23 $(25,5 \%)$ transtibiais e $34(37,8 \%)$ no pé.

\section{Discussão}

A amputação de membros tem uma incidência mundial de mais de 1 milhão ao ano. Este número, ao mesmo tempo em que tende a reduzir-se significativamente se realizados a prevenção e o tratamento precoce correto ${ }^{17}$, tende a aumentar devido ao aumento da expectativa de vida ${ }^{20}$, que se correlaciona com: uma maior prevalência das síndromes plurimetabólicas (DM, dislipidemias, obesidade, etc.), menor qualidade de vida (alimentação incorreta, sedentarismo, alto nível de estresse, abuso de substâncias nocivas, como álcool e tabaco) e maior risco de doenças crônicas e sistêmicas (doenças cardiovasculares, pneumopatias, neoplasias, hipertensão arterial, nefropatias, etc. $)^{21}$.

Estudos que abordam a incidência e o perfil dos pacientes submetidos às amputações se tornam de extrema importância frente a esse cenário mundial, e 
apesar de tudo ainda poucos o fazem ${ }^{2}$. Por isso foi realizado este trabalho, já que, independente de ser uma seqüela pós-trauma ou de doença, definitivamente a amputação é percebida como uma mutilação e afeta a vida de qualquer pessoa ${ }^{6}$.

O modelo, retrospectivo e descritivo, visa determinar a incidência das amputações realizadas no referido hospital após a implantação do serviço de cirurgia vascular, levando em consideração o trauma ou a doença vascular como indicação para as operações, semelhante ao trabalho de Carmona et al. ${ }^{2}$, no qual a causa predominante foi a insuficiência vascular periférica.

Houve dificuldade na coleta de dados dos prontuários, o que é considerado por Almeida Filho \& Rouquayrol $^{22}$ como inerente aos estudos retrospectivos, indicando a necessidade de um grupo controle, mas com a dificuldade de determinar por que certo indivíduo seria alocado em um ou outro grupo e com a vantagem de ser um trabalho passível de ser realizado em curto prazo e também de ser menos dispendioso.

Pela falta de registro, não foi possível determinar o grau de escolaridade em 65 prontuários, mas pôde-se observar que, na amostra restante (46 pacientes), o número de analfabetos foi alto (41\%). Esta é uma informação compatível com o tipo de população atendida pelo Sistema Único de Saúde (SUS) no Paraná ${ }^{23}$. Nessa população, a ocorrência de amputações geralmente é mais alta, pois, para sua prevenção, o aprendizado dos cuidados individuais com os membros torna-se mais difícil, apesar de sua importância. Além disso, sabe-se que essa população é altamente dependente da Atenção Primária de Saúde, programa ainda com grandes falhas em nosso país ${ }^{24}$.

$\mathrm{Na}$ avaliação da amostra, foi observada uma diminuição dos procedimentos relacionados à causa vascular a cada ano, demonstrada pelo tempo de cada intervenção em relação ao início da pesquisa. Na implantação do serviço e pelo fato de ser um hospital público, houve muitos atendimentos de pacientes que há tempos buscavam tratamento, e por falta de condições econômicas este não era possível, levando ao agravamento da doença, que evoluía para um quadro irreversível, culminando com amputação. Após a resolução desses casos e da padronização do atendimento do ponto de vista vascular, foi ocorrendo a diminuição dos casos de amputação.

De qualquer forma, esta é uma tendência mundial, já que a prevenção da amputação é um objetivo da saúde pública do mundo inteiro, principalmente dando-se uma atenção maior à associação de doenças sistêmicas mais prevalentes $^{2,25}$.

A análise do fator causal trauma mostrou-se ser muito relevante e de grande impacto, pois a maioria era uma população jovem, do sexo masculino e, segundo Meirelles, na época mais produtiva da vida ${ }^{26}$. Sabendo-se que as causas mais comuns de amputação na infância estão relacionadas ao trauma em pré-escolares e escolares $^{27}$, nesse grupo havia um paciente de 4 anos submetido a uma amputação menor (pé), sendo esta devido a um esmagamento da extremidade, com perda óssea e de tecidos moles, de forma irreparável.

As pessoas com mais de 50 anos corresponderam a $82,8 \%$, período em que as doenças associadas ao risco de uma amputação são mais prevalentes ${ }^{28}$. A média de idade foi menor para os homens, coincidindo com dados encontrados em outras pesquisas ${ }^{2}$, mas, apesar disso, houve um aumento no índice de operações quanto maior a idade, semelhante a outros estudos ${ }^{2,29}$.

O predomínio do sexo masculino, com uma relação de 2,4:1, também foi semelhante a dados da literatura ${ }^{15}$.

Apesar do pequeno número da amostra considerada quanto ao nível de escolaridade, foi observado pelo teste de Fisher que não houve associação significante entre sexo e escolaridade (Tabela $1, \mathrm{p}=0,177$ ), mas no sexo feminino houve um menor número de amputações, semelhante aos resultados do trabalho de Bergamini ${ }^{23}$.

O perfil de predomínio da população com baixo nível socioeconômico e baixa escolaridade é um erro comum nesse tipo de pesquisa observacional de hospitais terciários de atendimento exclusivo do SUS, já que leva a um viés de seleção, podendo levar a super ou subestimação de certos fatores de risco, assim como prevalência e incidência em relação à população total ${ }^{22}$, fato este que deve ter ocorrido nesta pesquisa.

Foi observada uma importante associação entre a insuficiência vascular e fatores associados, sabendo-se 
que todos já têm sua relação estabelecida com o aumento do risco de evolução para a amputação, sendo o DM um dos principais agravantes ${ }^{2}$, pois é um fator que comprovadamente aumenta o risco de insuficiência vascular, elevando a chance de resultar em uma úlcera de MMII ou até amputações ${ }^{15,21,25}$, porém não foi observada associação significante entre o DM e os procedimentos realizados nesta amostra pelo teste qui-quadrado (Tabela 2, p = 0,551).

A HAS, isoladamente, já é considerada um risco, pois uma das suas conseqüências em longo prazo é a úlcera hipertensiva, a qual pode evoluir para infecção e amputação ${ }^{29,30}$; mas, neste trabalho, semelhante ao DM, também não houve associação significante entre a HAS e os procedimentos realizados, (desbridamentos e amputações), demonstrado pelo teste qui-quadrado (Tabela $3, \mathrm{p}=0,380)$.

O tabagismo e o risco aumentado de amputação são bem documentados, especialmente se em conjunto com o DM. É fato que no pé diabético de um fumante as chances de uma amputação são maiores, apesar de que, se o paciente parar com o hábito de fumar, consegue diminuir esse risco com o tempo ${ }^{21,31}$. Nesta pesquisa, com registro de apenas 83 pacientes quanto a esse hábito, houve presença de $59 \%$ de tabagistas e ex-tabagistas, e pelo teste de Fisher (Tabela 4, p = 1,000) não foi demonstrada relação significante entre o hábito e os procedimentos cirúrgicos, porém deve-se ressaltar a importância da prevenção desse hábito, apesar de o número de programas para dependentes do fumo ainda ser pequeno e não estar disponível ou não ser oferecido para todos ${ }^{32}$.

A relação precisa entre os fatores associados e a insuficiência vascular não pode ser calculada, já que os fatores, quando em concomitância, causam os erros de confusão ${ }^{22,33}$.

Os níveis séricos de creatinina com valores limítrofes superiores ou alterados podem revelar injúria tecidual e/ou componente renal pela doença básica ${ }^{34}$, mas neste trabalho a maioria dos pacientes $(76 \%)$ apresentava creatinina menor que $1,4 \mathrm{mg} / \mathrm{dL}$.

Outros exames de maior complexidade, como angiografia e ultra-som vascular com Doppler, raramente foram realizados, sendo difícil discutir algo a mais sobre isso.

As características das intervenções mostraram um maior número de amputações $(84,5 \%)$ do que desbridamentos $(15,5 \%)$, ocorrido talvez por ser início de atendimento do serviço de cirurgia vascular em um hospital de atendimento ao SUS de sua região; assim, grande parte dos casos, ao chegar, já estava em fase avançada, significando amputação e não mais somente desbridamento cirúrgico para tratamento. Segundo Almeida Filho ${ }^{22}$ e Sackett et al. ${ }^{33}$, este é um exemplo de viés de seleção comum nesse tipo de estudo.

Os protocolos de investigação clínica devem apontar ações de educação e assistência na área, como um bom controle metabólico, boa aderência aos tratamentos clínicos, boa integridade cutânea (higiene adequada, insensibilidade nos pés detectada por traumas com o uso de calçados inadequados e outros objetos, corte das unhas, onicomicoses e onicocriptoses tratadas corretamente, profissionais treinados para retirada de calos plantares evitando acidentes, etc.), identificação precoce de lesões neuroisquêmicas e sinais súbitos de isquemia periférica ${ }^{20,35,36}$.

Todas são medidas passíveis de se alcançar com os recursos que se tem atualmente, cujas conseqüências teriam um grande impacto para a prevenção dessas incapacidades, extremamente onerosas e que levam a problemas físicos, mentais e sociais irreversíveis ${ }^{37}$, mas nos grandes centros urbanos, em que pese a maior disponibilidade de oferta de serviços de saúde, particularmente os de média e alta complexidade, observam-se barreiras ao acesso e oferta de ações básicas ${ }^{24}$, sendo estimado que, com essas medidas e pela presença de uma equipe multidisciplinar treinada, pode-se diminuir em 45 a 85\% as amputações ${ }^{25}$.

A equipe para o atendimento deve ser integrada por cirurgião vascular, ortopedista, endocrinologista, enfermeira especializada nessa área, podólogo e uma equipe $\operatorname{administrativa~}^{25}$.

\section{Conclusão}

Este trabalho permite inferir que a incidência de amputação de MMII foi maior no sexo masculino, na 
faixa etária de 51 a 70 anos, teve como principal causa a DAOP e apresentou redução progressiva.

\section{Referências}

1. De Luccia N. Amputação e reconstrução nas doenças vasculares e no pé diabético. Rio de Janeiro: Revinter; 2006. p. 248.

2. Carmona GA, Hoffmeyer P, Herrmann FR, et al. Major lower limb amputations in the elderly observed over ten years: the role of diabetes and peripheral arterial disease. Diabetes Metab. 2005;31:449-54.

3. Chen SY, Chie WC, Lan C, Lin MC, Lai JS, Lien IN. Rates and characteristics of lower limb amputations in Taiwan, 1997. Prosthet Orth Int. 2002;26:7-14.

4. Moulik PK, Mtonga R, Gill GV. Amputation and mortality in new-onset diabetic foot ulcers stratified by etiology. Diabetes Care. 2003;26:491-4

5. Dangelser G, Besson S, Gatina JH, Blicklé JF. Amputations among diabetics in Reunion Island. Diabetes Metab. 2003;29:628-34.

6. Stiegler H. [Diabetic foot syndrome]. Herz. 2004;29:104-15.

7. Peden M, McGee K, Sharma G. The injury chart book: a graphical overview of the global burden of injuries. Geneva: World Health Organization; 2002.

8. Peden M, McGee K, Krug E. Injury: a leading cause of the global burden of disease, 2000. Geneva: World Health Organization; 2002.

9. Medeiros CAF, Landim RM, Castro NA, et al. Condutas no trauma penetrante da artéria axilar. J Vasc Bras. 2003;2:225-8.

10. Shalabi R, Al Amri Y, Khoujah E. Vascular injuries of upper extremity. J Vasc Bras. 2006;5:271-6.

11. Costa CA, Cabral PH, Pinto PL, Pinheiro Jr MJC, Santos NJN, Birolini D. Initial experience with hand replantation after traumatic amputation in a nonspecialized center. J Vasc Bras. 2006;5:321-4.

12. Araújo GM, Mathias SB, Felipe Jr G. Dados epidemiológicos. In: Murilo R, Brito CJ, Vergara E, Meirelles S. Trauma vascular. Rio de Janeiro: Revinter; 2006. p. 74-82.

13. Campos-Christo SF, Miguel EV, Costa-Val R, Teixeira ELC. Amputação primária no trauma: indicações e aspectos legais. In: Murilo R, Brito CJ, Vergara E, Meirelles S. Trauma vascular. Rio de Janeiro: Revinter; 2006. p. 153-60.

14. Holcomb JB, Jenkins D, Rhee P, et al. Damage control resuscitation: directly addressing the early coagulopathy of trauma. J Trauma. 2007;62:307-10.

15. Sequeira FM, Martins AB. [Limb amputations carried out in hospitals of the national health service in the years from 1990 to 1993]. Acta Med Port. 1996;9(9-7):207-10.

16. Van Damme H, Limet R. Le pied diabétique. Rev Med Liege. 2005;60:516-25.
17. Santos CAS, Nascimento PFT. Desbridamento e amputações. In: Pitta GBB, Castro AA, Burihan E, editores. Angiologia e cirurgia vascular: guia ilustrado. Maceió: UNCISAL/ECMAL \& LAVA; 2003. Disponível em: http://www.lava.med.br/livro.

18. Costa-Val R, Silva RCO, Nunes TA, Souza TKDP. O papel da oxigenoterapia hiperbárica na doença vascular periférica. J Vasc Bras. 2003;2:177-82.

19. Lima EB, Bernardes CHA, Martins ACG, Marcondes CM. O papel da oxigenoterapia hiperbárica no tratamento da gangrena gasosa clostridiana e da fasciite necrotizante. J Vasc Bras. 2003;2:220-4.

20. Regensteiner JG, Hiatt WR. Current medical therapies for patients with peripheral arterial disease: a critical review. Am J Med. 2002;112:49-57.

21. American Diabetes Association (ADA). Atualização: padrões e recomendações, patologias associadas. Diabetes Clin. 2000;4:118-36.

22. Almeida Filho N, Rouquayrol MZ. Estudo de casos. In: Almeida Filho N, Rouquayrol MZ. Introdução a epidemiologia. $3^{\text {a }}$ ed. Rio de Janeiro: Medsi; 2002. p. 196-205, 215-231.

23. Bergamini R. Um estudo sobre a população brasileira no século XX - Fonte IBGE, Revista Eletrônica Desemprego Zero, outubro, 2000. Disponível em: http:// www.desempregozero.org.br/artigos/um_estudo_sobre_a_ populacao_brasileira_no_seculo_xx_fonte_ibge.php.

24. Assis MMA, Cerqueira EM, Nascimento MAA, Santos AM, Jesus WLA. Atenção primária à saúde e sua articulação com a estratégia saúde da família: construção política, metodológica e prática. Revista de APS. 2007;10:189-99. Disponível em: http://www.nates.ufjf.br/novo/revista/ v010n2.htm.

25. Krishnan ST, Rayman G. Reducing amputations in "at risk foot". Indian J Med Res. 2005;122:368-70.

26. Meirelles SSL. Traumatismo arterial de membros inferiores. In: Pitta GBB, Castro AA, Burihan E, editores. Angiologia e cirurgia vascular: guia ilustrado. Maceió: UNCISAL/ECMAL \& LAVA; 2003. Disponível em: http://www.lava.med.br/livro.

27. Belangero WD, Livani B, Angelini AJ, Davitt M. Amputaçäo dos membros inferiores na criança. Relato e experiência em 21 casos. Acta Bras Ortop. 2001;9:6-10.

28. Diogo MJD. A dinâmica dependência-autonomia em idosos submetidos à amputação de membros inferiores. Rev Latino-Am Enfer. 1997;5:59-64.

29. De Godoy JM, De Godoy MF, Batigalia F, Trávolo AR, Monteiro EH. Lower-extremity amputation: a 6-year follow-up study in Brazil. J Orthop Surg (Hong Kong). 2005;13:164-6.

30. Graves JW, Morris JC, Sheps SG. Martorell's hypertensive leg ulcer: case report and concise review of the literature. J Hum Hypertens. 2001;15:279-83.

31. Deluchi Levene NA. Ulcera hipertensiva de Martorell. Rev Argent Cirug. 1991;61:209-10.

32. Schaan BD, Mandelli NCB. Conduta na doença arterial periférica em pacientes diabéticos. Rev Soc Cardiol RGS. 2004. Disponível em: http://sociedades.cardiol.br/sbc-rs/revista/ 2004/02/artigo02.pdf. 
33. Gamba MA, Gotlieb SLD, Bergamaschi DP, Vianna LAC. Amputações de extremidades inferiores por diabetes mellitus: estudo caso-controle. Rev Saude Publica. 2004;38:399-404.

34. Sackett DL, Strauss SE, Richardson WS, Rosenberg W, Haynes RB. Medicina baseada em evidência: prática e ensino. $2^{\mathrm{a}}$ ed. Porto Alegre: Artmed; 2003. p.40-92.

35. Gross JL, Silveiro SP, Canani LH, Friedman R, Leitão CB, de Azevedo MJ. Diabetic nephropathy and cardiac disease. Arq Bras Endocrinol Metabol. 2007;5:244-56.

36. American Diabetes Association. Consensus development conference on diabetic foot wound care. Diabetes Care. 1999;22:1354-60.
37. Sheahan MG, Hamdan AD, Veraldi JR, et al. Lower extremity minor amputations: the roles of diabetes mellitus and timing of revascularization. J Vasc Surg. 2005;42:476-80.

Correspondência:

Amélia Cristina Seidel

Rua Dr. Gerardo Braga, 118, Jardim Vila Rica

CEP 87050-610 - Maringá, PR

Tel.: (44) 3026-7590

Fax: (44) 3225-0999

E-mail: seidel@wnet.com.br 